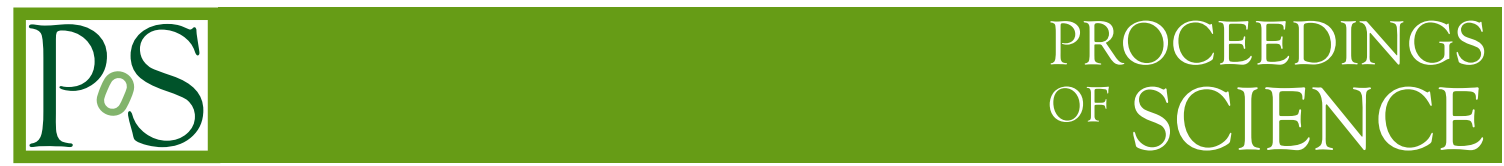

\title{
Neutrino physics from A to Z : Two lectures at Corfu
}

\author{
J.W.F. Valle \\ AHEP Group, Institut de Física Corpuscular - C.S.I.C./Universitat de València \\ Parc Cientific de Paterna. C/Catedratico José Beltrán, 2 E-46980 Paterna (València) - SPAIN \\ E-mail: valle@ific.uv.es, URL: http://astroparticles.es/
}

\begin{abstract}
Assuming basic familiarity with neutrino physics, I give a telegraphic and panoramic view of the current status and the main open challenges in the field. I also illustrate how the mechanism responsible for neutrino mass generation may shed light upon some of the current puzzles in particle physics as well as cosmology*
\end{abstract}

Proceedings of the Corfu Summer Institute 2016

"Summer School and Workshop on the Standard Model and Beyond" August 31 - September 12, 2016

Corfu, Greece

*This writeup corresponds to a slightly modified/updated version of the material actually presented at the School. 
A Introduction. The last few decades have seen a tremendous progress in particle physics and cosmology, the physics of the early universe. The discoveries of the Higgs boson at the Large Hadron Collider at CERN [1,2] and of neutrino oscillations [3,4] as a result of solar and atmospheric studies constitute major milestones in astroparticle physics which led to the 2012 and 2015 physics Nobel prizes. It is not an overstatement to say that the discovery of the Higgs boson has brought our field to euphoria. Some considered it as the last brick in the construction of the standard model. It is not, since in the Standard Model neutrinos have no mass, needed to account for the oscillation data [5]. More bricks are needed to fabricate neutrino mass. Indeed, as a key building block of the Standard Model, the properties of neutrinos may point us the way beyond the standard model [6]. However small the magnitude of their masses results to be, the electroweak breaking mechanism can be significantly affected by the presence of massive neutrinos, with potentially profound implications, e.g. for the consistency of the electroweak vacuum. Likewise neutrinos constitute an ideal cosmic messenger capable of exploring the earliest moments after the Big Bang. For example, establishing the existence of leptonic CP violation is an important goal in the agenda of upcoming oscillation experiments such as that of the DUNE proposal. Such a discovery would pave the way to elucidate one of the great cosmological puzzles i.e. the understanding the prevalence of matter over anti-matter in our universe.

On the other hand, the Standard Model does not include gravity. The first observation of gravitational waves by the LIGO Scientific Collaboration and Virgo Collaboration teams [7], have brought the ultimate need for the inclusion of gravity in our world picture more into the forefront than ever. Reconciling gravity and quantum mechanics is a formidable challenge that lies with us for over a century. Now the time seems to have come! There are, in addition, a variety of other, theoretical motivations for having beyond the Standard Model physics, such as understanding anomaly cancellation, unification of the forces, the consistency of the spontaneous symmetry breaking mechanism, including naturalness, stability and perturbativity. Unfortunately, other than the discovery of neutrino mass and some cosmological hints, the search for unambiguous signs of new phenomena in particle physics has so far been fruitless. In these lectures I will assume that you know the basics and focus on illustrating how the theory responsible for generating neutrino mass may also touch several of the above points.

B Neutrino probes. Neutrinos are tiny weakly interacting particles travelling close to the speed of light. They constitute one of the most ubiquitous particles in nature. Thanks to their weak interaction, neutrinos are excellent astrophysical messengers, probing the deep interior of the Sun or of a supernova. Likewise they probe the earliest instants of the universe, just after the Big Bang. Natural and artificial neutrino sources span about 20 orders of magnitude in energy, all the way from the abundant neutrinos produced in the Big-Bang to the ultra high energy cosmic ray neutrinos. The former are abundant, though currently undetectable because of their very low energy. The latter have much higher interaction rates, though their detection also constitutes a challenge thanks 
to the small fluxes. In between these extremes we have geoneutrinos, supernova neutrinos, as well as solar and atmospheric neutrinos.

C Solar and atmospheric neutrinos. Neutrinos are copiously produced in nuclear fusion reactions that power the Sun, while atmospheric neutrinos hit the Earth from all directions in the sky, produced by cosmic ray interaction in the upper atmosphere. These neutrinos are detected in gigantic underground detectors. In both cases there is a significant discrepancy between produced and detected neutrinos. The study of solar and atmospheric neutrinos wrote an important chapter in particle physics, leading to the discovery of neutrino oscillations $[3,4]$ and the physics Nobel prize in 2015 . The discovery of neutrino oscillation has been beautifully confirmed by earthbound experiments based at reactors and accelerators.
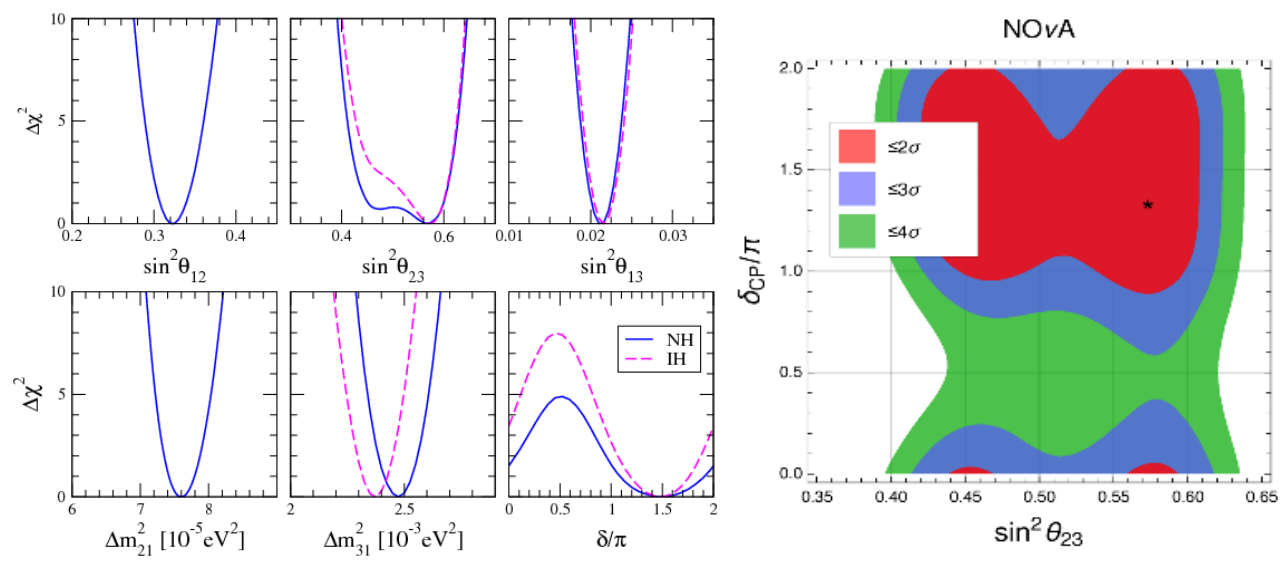

Figure 1: Left: Current status of oscillation parameters. Note the octant ambiguity in the atmospheric angle determination for $\mathrm{NH}$ and the very poor $\mathrm{CP}$ violating phase determination, according to the global fit [5]. Right: expected octant and CP sensitivity at the long baseline oscillation experiment NOvA, from [8].

D Reactors and accelerator neutrinos. All of these data are beautifully described by assuming that neutrinos undergo oscillations, a quantum mechanical phenomenon, as they propagate. The phenomenon is affected by the presence of matter, as it happens both in the interior of the Sun as well as in the Earth. Except for the atmospheric angle and CP violating phase, current neutrino oscillation experiments provide a good determination of the oscillation parameters, as seen in Fig. 1. Apart from the smallness of neutrino squared mass splittings, one thing that strikes the eye is the large values of the lepton mixing angles with respect to those that characterize the KobayashiMaskawa matrix. Resolving the atmospheric octant will require an improved measurement of the reactor angle $\theta_{13}$ [8]. The precision in the measurement of $\theta_{23}$ and $\delta_{C P}$ will be improved at the long baseline oscillation experiment NOvA and at the upcoming DUNE The bands correspond to the 2 , 3 , and $4 \sigma$ C.L uncertainties. Note that oscillations do not probe the absolute neutrino mass nor are they currently sensitive to the ordering of the neutrino states. 
E The absolute neutrino mass. Currently there are three realistic ways to probe the absolute neutrino mass: (i) measuring the shape of the end point of the spectrum in tritium beta decays, (ii) the search for neutrinoless double beta decay $\beta \beta_{0 v}$ as well as (iii) measurements of temperature anisotropies in the cosmic microwave background. Now we discuss (ii). Two-neutrino double-beta decay is the second-order weak interaction process by which two neutrons in a nucleus are converted to protons, plus two electrons plus two electron anti-neutrinos, $(A, Z) \rightarrow(A, Z+2)+2 e+2 v$. This very rare process has been detected in a few nuclei. It conserves lepton number. On the other hand $\beta \beta_{0 v}$ is a neutrinoless variety expected to occur if neutrinos are Majorana. Its amplitude is proportional to an effective mass parameter $\left\langle m_{\beta \beta}\right\rangle$ given in Fig. 2 as a function of the lightest neutrino mass. The dark shaded regions are generic predictions based on best-fit neutrino oscillation parameters for normal hierarchy $(\mathrm{NH})$ and inverted hierarchy (IH). The light shaded regions are the corresponding $3 \sigma$ ranges. The lowest horizontal band indicates the $90 \%$ C.L. upper limit on $\left\langle m_{\beta \beta}\right\rangle$ from KamLAND-Zen, using ${ }^{136} \mathrm{Xe}$. The upper bands give the sensitivities for other nuclei taking into account nuclear matrix element calculations [9]. The side-panel shows the corresponding limits for each nucleus as a function of the mass number. One sees a big experimental race to search

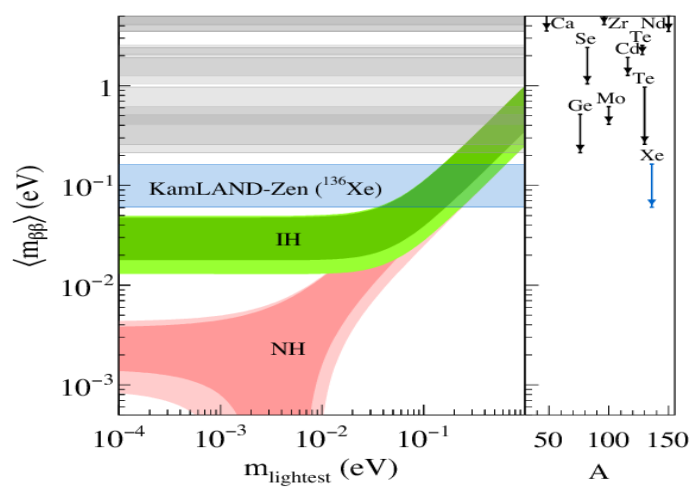

Figure 2: Status and prospects for $\beta \beta_{0 v}$ searches, from [9], see text.

$\beta \beta_{0 v}$ and possibly discover it! However, in the case of NH there can be destructive interference leading to a cancellation in the expected $\beta \beta_{0 v}$ rate. From this point of view it is very interesting to consider theories where the flavor structure is predicted in such a way that the cancellation is prevented. In such models there is a lower bound on the $\beta \beta_{0 v}$ decay rate. Examples are given in [10-13]. Note also that $\beta \beta_{0 v}$ can be induced by a short range mechanism, mediated by heavy states, through a variety of mechanisms [14]. In such case new signatures are expected at colliders such as the LHC $[15,16]$. The deep significance of $\beta \beta_{0 v}$ rests upon the fact that, irrespective of its origin, the observation of $\beta \beta_{0 v}$ implies lepton number violation and the Majorana nature of neutrinos $[17,18]$. This summarizes our brief summary of the experimental status of neutrino physics. For the corresponding references, see Refs. [3,4], [6,9] and references therein. Now we turn to theory, starting with the origin of neutrino mass and then moving to the structure of neutrino 
mixing and their theoretical origin.

F Weinberg dimension five operator. The origin of neutrino mass is one of the most well kept secrets of nature. A very general model independent approach was suggested by Weinberg, who noted that one can form a dimension five operator ${ }^{1}$, with the Standard Model lepton and Higgs doublets. This turns into a Majorana neutrino mass once the electroweak symmetry breaks through the nonzero vacuum expectation value (vev) of the Higgs doublet. The smallness of neutrino mass would be ascribed to the mass scale characterizing the $d=5$ operator $\mathscr{O}$, which was originally expected to be violated at high scale. However, the operator $\mathscr{O}$ may also be characterized by a small

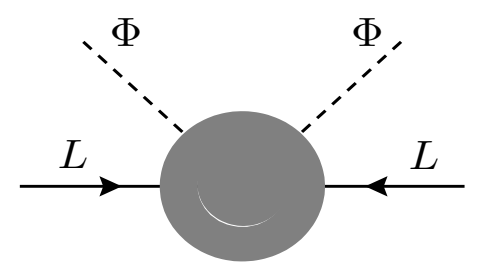

Figure 3: Dimension 5 operator leading to neutrino mass.

scale, so that in its absence the symmetry of the theory would increase, since lepton number would be recovered. This is a realization of t'Hooft's naturalness criterion. This is a generic argument. Nothing is known about the underlying mechanism that engenders this operator, its characteristic scale or its flavor structure. For that we need a theory. Various alternative theories may be classified by the way they generate the operator $\mathscr{O}$, with two broad sub-categories, namely seesaw and radiative schemes.

G Standard seesaw mechanism. This is by far the most popular approach to neutrino mass generation [6]. It assumes that the dimension five operator arises at tree level either through the exchange of new heavy right-handed neutrinos (type-I seesaw) or by the exchange of a triplet of scalars (type-II or triplet seesaw). Although the seesaw can be motivated by grand unified theories (GUTS) or by models with intermediate scales (e.g. Pati-Salam or Peccei-Quinn), the most general seesaw formulation is at the Standard Model level [20] ${ }^{2}$. In its original formulation, for example,
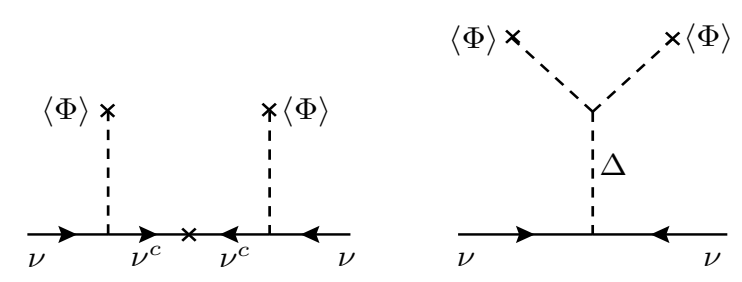

Figure 4: Type-I (Left) and Type-II (Right) seesaw mechanism.

\footnotetext{
${ }^{1}$ It is also possible to induce neutrino mass through higher order operators [19].

${ }^{2}$ It is curious that, in Ref. [20], the type-I/type-II naming was swapped with respect to what became later established.
} 
the type-I seesaw mechanism was thought to involve the exchange of heavy intermediate fermions, at a mass scale associated, say, with grand unification. Barring the the use of arbitrarily small Yukawa couplings to account for the small neutrino masses, there are no expected collider physics implications in this case. See, in contrast, discussion on low-scale seesaw, below.

H Low-scale seesaw mechanism and t'Hooft's naturalness. One should realize that the seesaw mechanism is not a model, rather a general framework for generating neutrino mass. As such, its general formulation clearly allows for "genuine" low-scale realizations ${ }^{3}$ so that when the coefficient of the operator $\mathscr{O}$ becomes zero the symmetry of the theory enhances as a result. Hence
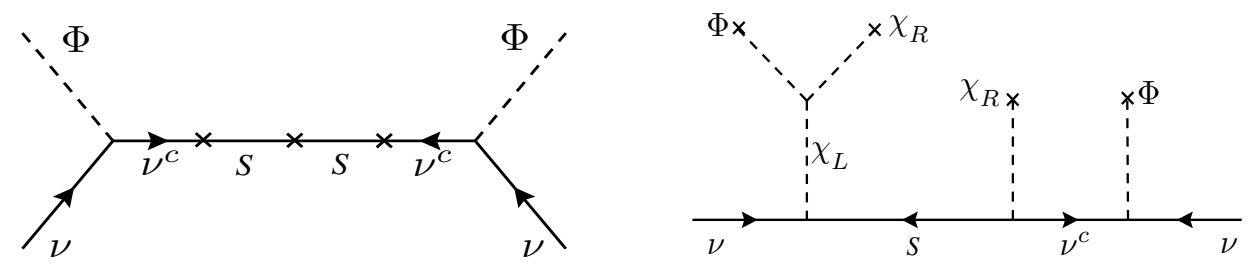

Figure 5: Low-scale seesaw mechanism: inverse (left) and linear (right) realizations.

there is, in this case, no need for the accompanying physics to live at a large scale. This is the theoretical basis of the low scale seesaw mechanism. There are two variants, namely linear and inverse seesaw. They are currently very popular as they open the possibility of direct production of the neutrino mass generation messengers at colliders experiments. In the presence of a new gauge portal these lead to lepton flavour violation signatures at colliders such as the LHC or the future proposals such as ILC/CLIC $[15,16]$.

I Dirac seesaw mechanism. Almost forty years after the seesaw idea first appeared, we have developed a full conceptual description of both Type-I as well as Type-II seesaw siblings of the seesaw mechanism for the case of Dirac neutrinos. In order to ensure the Dirac nature of neutrinos, two states associated to "left" and "right" are needed and, moreover, some extra symmetry principle is needed for "Diracness". For example, in the type-II case the smallness of neutrino mass

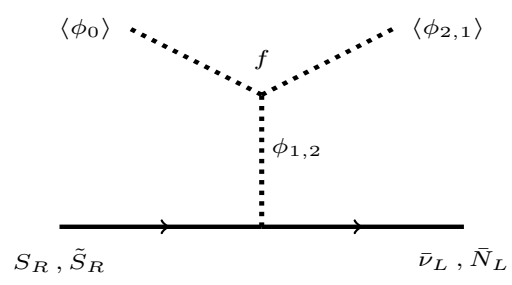

Figure 6: Type-II Dirac seesaw mechanism.

follows from a parameter whose absence enhances the symmetry of the theory, hence natural in

\footnotetext{
${ }^{3}$ Genuine low-scale means that tiny neutrino masses do not require arbitrarily small parameters, such as Yukawa couplings. The seesaw scale in any high-scale type-I seesaw can be made arbitrarily low by lowering the Dirac Yukawas.
} 
t'Hooft's sense. Neutrino mass generation may result from the spontaneous breaking of a global $\mathrm{U}(1)$ symmetry, leading to a physical Nambu-Goldstone boson - a Dirac sibling of the majoron - we call Diracon. Like the majoron case also the Diracon couplings are severely restricted by bounds from stellar cooling rates in astrophysics. Although stringent, these are consistent with possibly significant invisible Higgs decays to Diracons, well constrained by studies at colliders such as the LHC.

J Radiative mechanisms. These constitute an interesting alternative to generate $\mathscr{O}$ without the need to invoke physics at inaccessible mass scales [21]. These typically involve new scalar

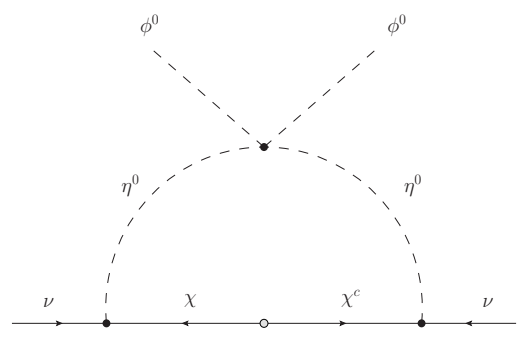

Figure 7: Dark matter as messenger of radiative neutrino mass generation [22,23].

bosons. A specially attractive example are the scotogenic models [24], interesting because they naturally incorporate dark matter. The latter emerges as a messenger of neutrino mass generation. The model invokes a $Z_{2}$ symmetry ensuring the radiative nature of neutrino mass and, at the same time, also stabilizing dark matter. A phenomenological richer realization of Ma's original idea has been proposed and studied in [22,23]. It is also possible that, in extensions of the electroweak gauge symmetry such as $\mathrm{SU}(3)_{\mathrm{C}} \otimes \mathrm{SU}(3)_{\mathrm{L}} \otimes \mathrm{U}(1)_{\mathrm{X}}$ schemes, neutrino masses may be induced radiatively as a result of the exchange of new gauge bosons [25].

K The flavor problem. The pattern of charged fermion masses is very strange, spanning about six orders of magnitude between the electron mass and the top quark mass. This is just one aspect of the flavor problem. Another one is the observed disparity between quark and lepton mixings. In fact, as seen above, the smallest of the lepton mixing angles, i.e. the "reactor" angle $\theta_{13}$, is similar to the largest of the quark mixing angle, namely the Cabibbo angle. In fact this may well be a subtle message nature is conveying to us. For example there may be a symmetry rationale in which the Cabibbo angle acts as a universal seed for all quark and lepton mixings [26-28] Also the magnitudes of the lepton mixing angles do not seem arbitrary parameters. The way we face the challenge of bringing some rationale to the observed pattern of fermion masses and mixings is through the use of symmetry approaches. In this context let us mention the interesting case of non-Abelian flavor symmetries. 
L Flavor-dependent $\mathbf{b}-\tau$ unification. As an example we mention here a successful flavor generalization of the $b-\tau$ unification formula characteristic of minimal SU(5), namely,

$$
\frac{m_{b}}{\sqrt{m_{d} m_{s}}}=\frac{m_{\tau}}{\sqrt{m_{e} m_{\mu}}}
$$

This relation holds approximately in a number of flavor models based on the $A_{4}[12,29,30]$ and $T_{7}$ [13] symmetries. This formula is a consistent, and fairly stable, generalization of the conventional SU(5) prediction, showing how one can relate quark and lepton masses without the need for grand-unification.

M BMV model as prototype flavor model. We now turn to the minimal flavor scheme proposed in [31], we call it BMV, for short. The model adopts A4, the smallest non-Abelian symmetry group with three-dimensional irreps where the three families of leptons can fit nicely. Valid at some high-energy scale, the flavor symmetry naturally leads to degenerate neutrino masses and hence to a sizeable rate for neutrinoless double beta decay. Realistic neutrino mass splittings are then induced by renormalization group evolution with threshold corrections. Assuming CP invariance the atmospheric mixing is predicted to be maximal, $\theta_{23}=\pi / 4$, and the reactor mixing $\theta_{13}$ is predicted to vanish ${ }^{4}$. Note that lepton flavour violation processes such as $\tau \rightarrow \mu \gamma$ are expected to lie within reach of upcoming experiments [32]. However, given the reactor results, e.g. from Daya-Bay, the prediction $\theta_{13}=0$ needs to be corrected.

(N) Oscillation prediction in revamped BMV. Generalizing BMV is simple and leads to nontrivial results. One finds that not only $\theta_{13}$ is generated, and hence $\mathrm{CP}$ violation in oscillations, but also a departure from maximality in the atmospheric mixing $\theta_{23}$. Moreover, that these parameters are correlated as shown in Fig. 8, left panel. The green region is the theory prediction, while the narrow dark bands are $1 \sigma$ and the broad light band corresponds to $3 \sigma$, according to the global fit [5].
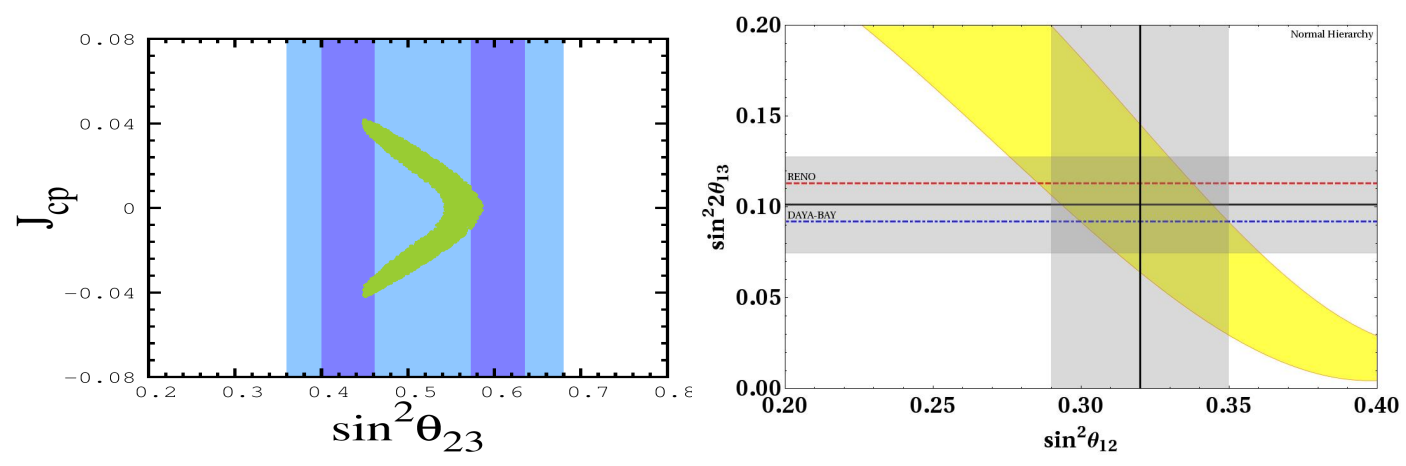

Figure 8: Left: $\mathrm{CP}$ versus atmospheric angle correlation predicted in revamped BMV model [33]. Right: Correlation between reactor and solar mixing in $\Delta(54)$ flavor model [34]. Bands indicate oscillation results.

\footnotetext{
${ }^{4}$ In the presence of CP violation $\theta_{13}$ is arbitrary and CP must be maximally violated in neutrino oscillations ggg
} 
In fact, very often the predictions one obtains from flavor models are expressed as correlations between oscillation parameters, for example between the solar angle $\theta_{12}$ and the reactor $\theta_{13}$, as seen in the right panel of Fig. 8. There is a huge literature on flavor model building, many symmetries can be used, one-by-one on a trial-and-error basis [35-37]. It is fun, but can be rather tedious.

(O) Residual CP symmetries. Before closing this discussion, let me comment on an alternative model-independent approach is to exploit symmetries of the neutrino mass matrix, such as generalized CP symmetries, irrespective of how exactly they emerge within a particular model. This way one can study flavor predictions in a model-independent way. The prototype case is mu-tau parity symmetry and generalizations thereof [38]. For example, a particular prediction in the $\delta_{C P}$ versus $\theta_{23}$ plane has been studied in [39] and many more were analysed in [40]. These may be eventually tested by the new generation of neutrino oscillation experiments, such as NOvA, DUNE and T2HK.

(P) Gauge coupling unification. Within the Standard Model gauge coupling unification is a "near-miss": when extrapolated to high energies from their measured low-energy values, the three gauge couplings almost meet together, but not quite. Gauge coupling unification constitutes, indeed, an attractive argument suggesting the existence of physics beyond the Standard Model. What

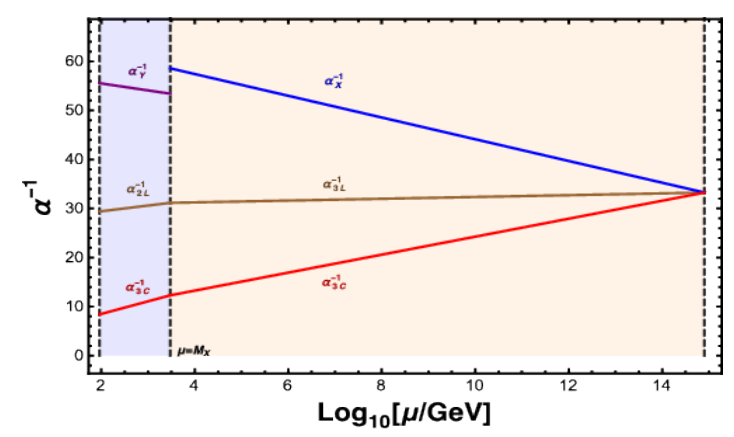

Figure 9: Gauge coupling unification may be triggered by the physics inducing small neutrino mass [41,42].

makes the gauge couplings unify? Common frameworks to provide plausible answers to this question are (i) grand unification and (ii) $\mathrm{TeV}$ scale supersymmetry. However, neither of their characteristic predictions, such as proton decay or the existence of supersymmetric states, have so far been vindicated experimentally. A logical possibility is that gauge coupling unification is a consequence of the same mechanism responsible for small neutrino mass generation, as recently proposed [41]. This is illustrated in Fig. 9. The model employs an extended $\mathrm{SU}(3)_{\mathrm{C}} \otimes \mathrm{SU}(3)_{\mathrm{L}} \otimes \mathrm{U}(1)_{\mathrm{X}}$ electroweak gauge structure, in the presence of a set of leptonic octets, directly involved in neutrino mass generation [42]. Possible embeddings probably require an F-theory GUT setup [43].

Q What and where is the new physics? So far there has been no hint of supersymmetry nor for any unexpected signature. Given the lack of striking new results from the LHC a burning question 
is what is the expected profile and scale of the new physics? Can one expect any "oasis" at the energies currently available at the LHC? Here we note that the historic discovery of the Higgs boson may suggest that it is only the first of a family associated, perhaps, to symmetry breaking patterns of more mundane extensions of the $\mathrm{SU}(3)_{\mathrm{C}} \otimes \mathrm{SU}(2)_{\mathrm{L}} \otimes \mathrm{U}(1)_{\mathrm{Y}}$ gauge group. There are motivations for extended gauge structures. For example left-right symmetry, which has as attractive feature the fact that it elevates parity to the status of a spontaneously broken symmetry, associated to the seesaw mechanism. Likewise, there are chiral Standard Model extensions whose quantum consistency requires exactly three families of fermions. Which pattern of new physics should be expected at current and upcoming accelerator experiments? Similarly important, On what grounds can one choose the preferred one?

\section{R Parity non-conservation, the number of families and new gauge bosons.}

In order to provide a framework to answer the above questions one may consider some of the open conceptual issues in weak interaction theory. For example, (i) What is the role of parity nonconservation?, (ii) what is the origin of neutrino mass? or (iii) Is there a rationale for having just three families of quarks and leptons? Here we consider these three features as possible ways to establish a criterion for choosing the Standard Model extension. They can be reconciled in a left-right

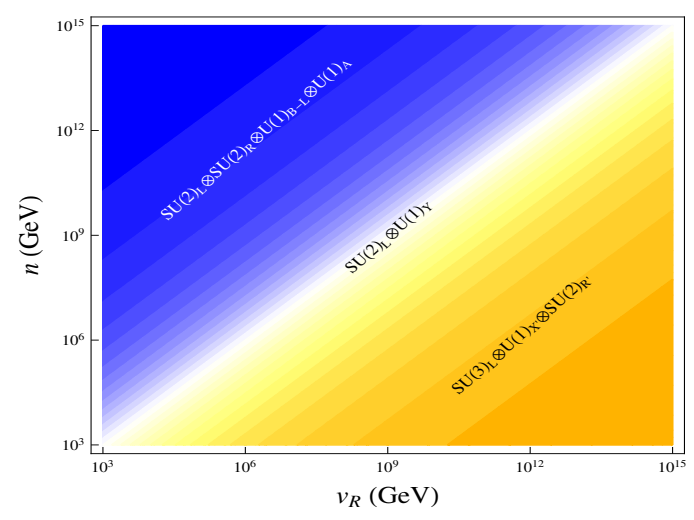

Figure 10: Dynamically determining the new physics profile through the vev ratio $n / v_{R}$ [44].

symmetric "mother" theory based on the $\mathrm{SU}(3)_{\mathrm{C}} \otimes \mathrm{SU}(3)_{\mathrm{L}} \otimes \mathrm{SU}(3)_{\mathrm{R}} \otimes \mathrm{U}(1)_{\mathrm{X}}$ gauge group [44]. Indeed, the mother theory is a realistic manifestly left-right symmetric setup, requiring the number of families to match the number of colors in order to achieve quantum consistency. Neutrino masses arise either from the canonical seesaw mechanism [44] or from a low-scale seesaw picture [45]. Small neutrino mass correlates with the observed V-A nature of the weak force. Depending on the symmetry breaking path to the Standard Model one recovers as the next step towards new physics either a conventional $\mathrm{SU}(3) \otimes \mathrm{SU}(2)_{\mathrm{L}} \otimes \mathrm{SU}(2)_{\mathrm{R}} \otimes \mathrm{U}(1)_{\mathrm{B}-\mathrm{L}}$ scenario or one based on a manifestly chiral extension of the electroweak symmetry based on $\mathrm{SU}(3)_{\mathrm{C}} \otimes \mathrm{SU}(3)_{\mathrm{L}} \otimes \mathrm{U}(1)_{\mathrm{X}}$ in which the number of families is fixed through anomaly cancellation. If light enough, the resulting $Z^{\prime}$ gauge bosons can be produced at the LHC, providing a production portal for the right-handed neutrinos, 
whose decays would engender lepton flavour violation processes [46]. On the other hand, its flavor changing interactions would also affect the K, D and B neutral meson systems [47]. The interplay of the $B_{d}^{0}-\bar{B}_{d}^{0}$ mass difference (left panel in Fig. 11) and the LHC dilepton mass constraints for two different parametrizations of the quark mixing matrix is illustrated in right panel in Fig. 11. In
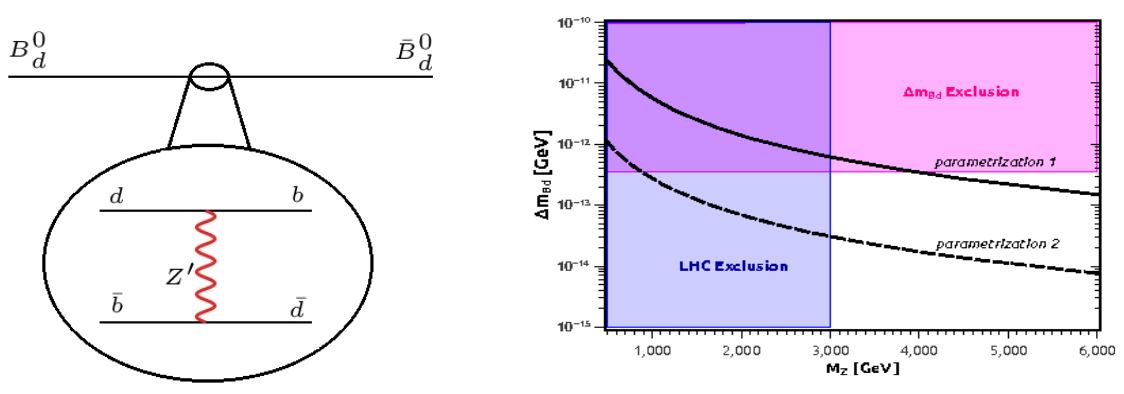

Figure 11: Feynman diagrams relevant for dilepton production at the LHC and the $B_{d}^{0}-\bar{B}_{d}^{0}$ mass difference in minimal low-scale $\mathrm{SU}(3)_{\mathrm{C}} \otimes \mathrm{SU}(3)_{\mathrm{L}} \otimes \mathrm{U}(1)_{\mathrm{X}}$ model.
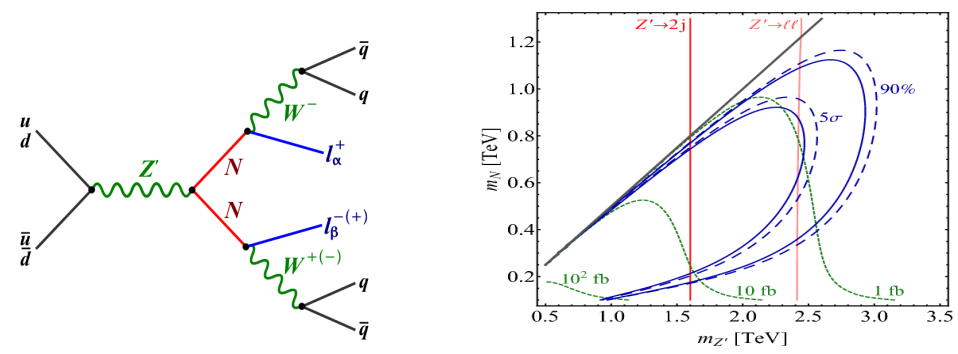

Figure 12: 3-3-1 model.

such scenario the right-handed neutrino messengers of neutrino mass generation may be produced via a $Z^{\prime}$ portal and can decay via small flavor violating couplings. This leads to lepton flavour violation at the LHC $[15,16]$, whose the rates are unsuppressed despite unobservably small $\mu \rightarrow e+\gamma$ gamma rates [46].

(S) Neutrino mass generation The historic discovery of the $125 \mathrm{GeV}$ Higgs boson may suggest that it is only the tip of the iceberg. Indeed, it is likely that this Higgs is just the first of a family, there could be others associated with the breaking of symmetries such as lepton number. For example extra scalar multiplets beyond those in the $\mathrm{SU}(3)_{\mathrm{C}} \otimes \mathrm{SU}(2)_{\mathrm{L}} \otimes \mathrm{U}(1)_{\mathrm{Y}}$ theory, such as singlet and triplet Higgses, are used to generate small neutrino mass in the seesaw mechanism. If ungauged, spontaneous lepton number violation implies the existence of a physical Nambu-Goldstone boson - the majoron $[48,49]$. The good measurement of the invisible $Z^{0}$ decay width implies that the majoron must be mainly singlet. If the associated scale is relatively low, such singlet "majoron model" naturally implies potentially observable rates for invisible Higgs decays [50], leading to missing momentum signatures at accelerators [51,52]. Such are now well constrained by LEP [53] 
and LHC experiments [54].

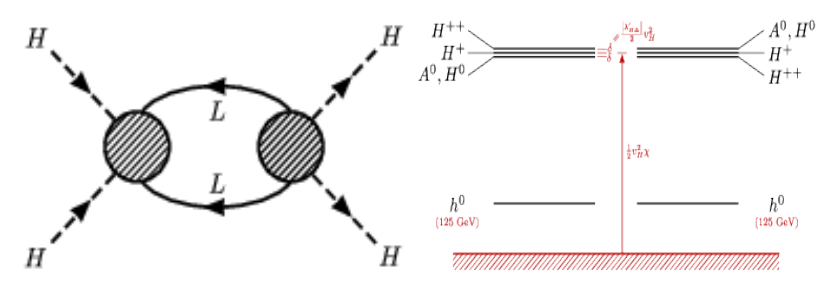

Figure 13: Small neutrino mass, vacuum stability and perturbative electroweak breaking.

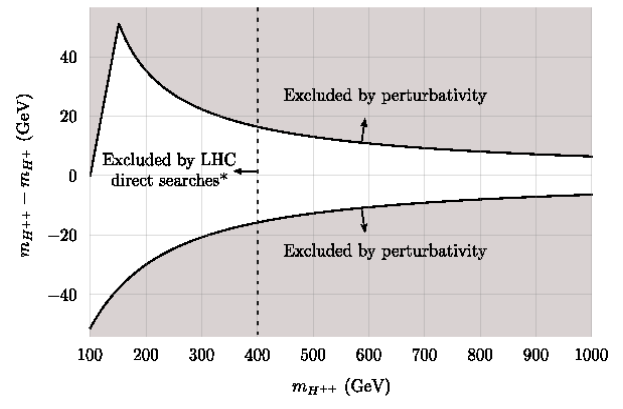

Figure 14: Schematic type-II seesaw Higgs spectrum.

T Neutrino mass generation and the consistency of the electroweak vacuum. We have just discussed that, if the smallness of neutrino mass is associated to a spontaneously broken symmetry, we need extra Higgs multiplets. Hence, in addition to the $\mathrm{SU}(3)_{\mathrm{C}} \otimes \mathrm{SU}(2)_{\mathrm{L}} \otimes \mathrm{U}(1)_{\mathrm{Y}}$ gauge invariance of the Standard Model we must also break such symmetry, such as lepton number, so as to account for naturally small neutrino mass. In the presence of extra "leptophilic" scalars the quartic coupling, whose positivity characterizes electroweak vacuum stability, gets new contributions. This naturally provides a mechanism to stabilize the theory's vacuum leading to a bounded-from-below potential energy. [55]. The mechanism may be qualitatively understood by "squaring" Weinberg' $\mathrm{s}$ operator, as indicated in Fig. 14. If we now evolve the theory with renormalization group equations all the way up to the Planck scale and require perturbativity to be maintained, then we find further constraints on electroweak breaking. For example, if a Higgs triplet is present, as in type-II seesaw, the mass splitting of its components is strongly restricted [56], leading to a "compressed" Higgs mass spectrum, as seen in the middle and right panels in Fig. 14. They give a schematic view of the scalar boson mass spectrum in the triplet seesaw model and one sees that the heavy scalars are nearly degenerate. There is a broad class of "neutrino motivated" extensions of the Standard Model Higgs sector, which provide interesting benchmark theories of electroweak breaking.

U How about Gravity? As a fundamental interaction of nature, gravity is described geometrically in Einstein's General Relativity. This is an elegant classical theory, not part of the Standard Model. Indeed, we still have not been able to develop a quantum theory of gravity. Unifying 
gravity with the quantum field theoretic Standard Model description of microphysics constitutes the biggest challenge in contemporary physics. The current approaches employ extra space-time dimensions either in the context of string theories or of warped geometries. Although both scenarios can be employed to provide frameworks for adding gravity, here we just consider the effective four-dimensional low energy theory that results from them after decoupling gravity. The question one may pose is whether the latter can lead to some predictions for neutrinos. For example, consider the extended $\mathrm{SU}(3)_{\mathrm{C}} \otimes \mathrm{SU}(3)_{\mathrm{L}} \otimes \mathrm{U}(1)_{\mathrm{X}}$ electroweak symmetry framework discussed in P. Although it can not easily be unified within the conventional field theory sense, it was shown that it admits a string completion within a quiver setup [57]. They constitute one of the most ubiquitous particles in nature. Thanks to their weak interaction, neutrinos are excellent astrophysical messengers, probing the deep interior of the Sun or of a supernova. Likewise they probe the earliest instants of the universe, just after the Big Bang. Natural and artificial neutrino sources span about 20 orders of magnitude in energy, all the way from the abundant neutrinos produced in the Big-Bang to the ultra high energy cosmic ray neutrinos. The former are abundant, though currently undetectable because of their very low energy. The latter have much higher interaction rates, though their detection also constitutes a challenge thanks to the small fluxes. One finds that lepton and baryon numbers are perturbatively conserved, so neutrinos are Dirac fermions. Moreover, consistent anomaly cancellation require extra "right-handed" neutrino-like states which lead to a natural realization of a novel, potentially low scale, type-II seesaw mechanism [58], as was illustrated in Section I, above.

V Neutrino predictions from Warped Standard Model. Randal and Sundrum [59] suggested a higher-dimensional mechanism to account for the hierarchy problem. The weak scale is generated from a large scale of order the Planck scale through an exponential hierarchy arising from the background metric, assumed to be a slice of AdS5 space-time. In principle this nice mechanism may be used to "explain" other mass hierarchies, such as those amongst the Standard Model fermions. Ref. [60] proposed a realistic five-dimensional warped Standard Model scenario with all fields propagating in the bulk, as illustrated in Fig. 15

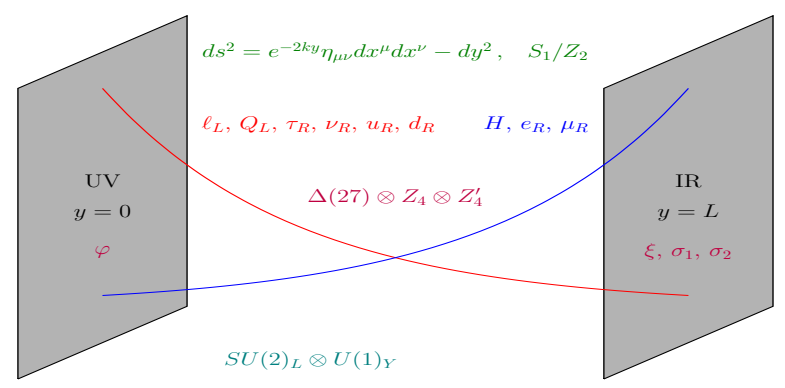

Figure 15: Basic structure of the warped model, showing the UV (IR) peaked nature of the fields, from [60].

Mass hierarchies are accounted for by judicious choices of the bulk mass parameters, while fermion 
mixing angles are restricted by a $\Delta(27)$ flavor symmetry broken on the branes by flavon fields. Note that, like the other fermions, here neutrinos are Dirac type. The flavor symmetry implies latter gives stringent predictions for the neutrino mixing parameters, and the Dirac CP violation phase, all described in terms of only two independent parameters at leading order. This leads to a correlation between $\mathrm{CP}$ violation and the atmospheric mixing parameter, in the same spirit as that in Fig. 8. These lead to predictions for the upcoming long baseline accelerator experiments T2K, NOvA and DUNE [61]. Note also that this Warped Standard Model gives a realistic CKM matrix [60].

X Neutrinos in Cosmology. According to the Big Bang the evolution of the universe started from a very hot and dense past and went through a number of phase transitions, dictated by the microphysics. In other words, the particle physics describing the interaction of the elementary constituents will determine how the universe evolves. As one of the most ubiquitous particles in nature, neutrinos play a key role in the evolution of the universe. Thanks to their weak interaction, they can probe the earliest epochs in the history of the universe, just after the Big Bang. In contrast, through optical means the universe can only be probed after the decoupling at 400,000 years or so [62]. Indeed, observations of temperature and polarization anisotropies of the Cosmic Microwave Background (CMB) enable us to obtain limits of the neutrino total mass. However, neutrinos can probe much earlier epochs, such as that associated with the breaking of the electroweak or higher symmetries. Indeed, it is precisely in association new physics at these scales that can address current puzzles in cosmology, such as dark matter, inflation and generation of the baryon asymmetry.

Z Majoron as warm decaying dark matter. One can formulate interesting new physics scenarios where the physics associated with neutrino mass generation can potentially reconcile at least some of the current cosmological puzzles as well. For example in the scheme suggested in [63] a single complex field is added whose vacuum expectation value breaks lepton number and generates neutrino mass through the seesaw mechanism, while the real part drives inflation and the imaginary part plays the role of metastable warm dark matter. Indeed this scenario has been shown to be consistent with restrictions from the CMB [64,65], and also to lead to potentially viable indirect detection by searching for X-ray lines $[66,67]$.

Acknowledgements: Supported by the Spanish grants FPA2014-58183-P, Multidark CSD200900064, SEV-2014-0398 (MINECO) and PROMETEOII/2014/084 (Generalitat Valenciana). I thank the school organizers for the warm hospitality and Carlos Vaquera for reading the manuscript.

\section{References}

[1] Aad, G. et al. Observation of a new particle in the search for the Standard Model Higgs boson with the ATLAS detector at the LHC. Phys.Lett. B716, 1-29 (2012). 1207 . 7214. 
[2] Chatrchyan, S. et al. Observation of a new boson at a mass of $125 \mathrm{GeV}$ with the CMS experiment at the LHC. Phys.Lett. B716, 30-61 (2012). 1207 . 7235.

[3] Kajita, T. Nobel Lecture: Discovery of atmospheric neutrino oscillations. Rev. Mod. Phys. 88, 030501 (2016).

[4] McDonald, A. B. Nobel Lecture: The Sudbury Neutrino Observatory: Observation of flavor change for solar neutrinos. Rev. Mod. Phys. 88, 030502 (2016).

[5] Forero, D., Tortola, M. \& Valle, J. W. F. Neutrino oscillations refitted. Phys.Rev. D90, 093006 (2014). 1405.7540 .

[6] Valle, J. W. \& Romao, J. C. Neutrinos in high energy and astroparticle physics (John Wiley \& Sons, 2015).

[7] Abbott, B. P. et al. Observation of gravitational waves from a binary black hole merger. Phys. Rev. Lett. 116, 061102 (2016). URL

https://1ink.aps.org/doi/10.1103/PhysRevLett.116.061102.

[8] Chatterjee, S. S., Pasquini, P. \& Valle, J. W. F. Probing atmospheric mixing and leptonic CP violation in current and future long baseline oscillation experiments (2017). 1702.03160.

[9] Gando, A. et al. Search for Majorana Neutrinos near the Inverted Mass Hierarchy Region with KamLAND-Zen. Phys. Rev. Lett. 117, 082503 (2016). [Addendum: Phys. Rev.

Lett.117,no.10,109903(2016)], 1605.02889.

[10] Dorame, L., Meloni, D., Morisi, S., Peinado, E. \& Valle, J. W. F. Constraining Neutrinoless Double Beta Decay. Nucl.Phys. B861, 259-270 (2012). 1111.5614.

[11] Dorame, L., Morisi, S., Peinado, E., Valle, J. W. F. \& Rojas, A. D. A new neutrino mass sum rule from inverse seesaw. Phys.Rev. D86, 056001 (2012). 1203.0155.

[12] King, S., Morisi, S., Peinado, E. \& Valle, J. W. F. Quark-Lepton Mass Relation in a Realistic A4 Extension of the Standard Model. Phys. Lett. B 724, 68-72 (2013). 1301.7065.

[13] Bonilla, C., Morisi, S., Peinado, E. \& Valle, J. W. F. Relating quarks and leptons with the $T_{7}$ flavour group. Phys. Lett. B742, 99-106 (2015). 1411.4883.

[14] Bonnet, F., Hirsch, M., Ota, T. \& Winter, W. Systematic decomposition of the neutrinoless double beta decay operator. JHEP 1303, 055 (2013). 1212. 3045.

[15] Aguilar-Saavedra, J., Deppisch, F., Kittel, O. \& Valle, J. W. F. Flavour in heavy neutrino searches at the LHC. Phys.Rev. D85, 091301 (2012). 1203.5998.

[16] Das, S., Deppisch, F., Kittel, O. \& Valle, J. W. F. Heavy Neutrinos and Lepton Flavour Violation in Left-Right Symmetric Models at the LHC. Phys.Rev. D86, 055006 (2012). 1206.0256.

[17] Schechter, J. \& Valle, J. Neutrinoless Double beta Decay in SU(2) x U(1) Theories. Phys.Rev. D25, 2951 (1982).

[18] Duerr, M., Lindner, M. \& Merle, A. On the Quantitative Impact of the Schechter-Valle Theorem. JHEP 1106, 091 (2011). 1105.0901. 
[19] Bonnet, F., Hernandez, D., Ota, T. \& Winter, W. Neutrino masses from higher than d=5 effective operators. JHEP 10, 076 (2009). 0907.3143.

[20] Schechter, J. \& Valle, J. W. F. Neutrino Masses in SU(2) x U(1) Theories. Phys.Rev. D22, 2227 (1980).

[21] Boucenna, S. M., Morisi, S. \& Valle, J. W. The low-scale approach to neutrino masses. Adv.High Energy Phys. 2014, 831598 (2014). 1404.3751.

[22] Hirsch, M. et al. WIMP dark matter as radiative neutrino mass messenger. JHEP 1310, 149 (2013). 1307.8134.

[23] Merle, A., Platscher, M., Rojas, N., Valle, J. W. F. \& Vicente, A. Consistency of WIMP Dark Matter as radiative neutrino mass messenger. JHEP 07, 013 (2016). 1603.05685.

[24] Ma, E. Verifiable radiative seesaw mechanism of neutrino mass and dark matter. Phys.Rev. D73, 077301 (2006). hep-ph/ 0601225.

[25] Boucenna, S. M., Morisi, S. \& Valle, J. W. F. Radiative neutrino mass in 3-3-1 scheme. Phys. Rev. D90, 013005 (2014). 1405.2332.

[26] Boucenna, S., Morisi, S., Tortola, M. \& Valle, J. W. F. Bi-large neutrino mixing and the Cabibbo angle. Phys.Rev. D86, 051301 (2012). 1206.2555.

[27] Ding, G.-J., Morisi, S. \& Valle, J. W. F. Bi-large Neutrino Mixing and Abelian Flavor Symmetry. Phys.Rev. D87,053013 (2013). 1211.6506.

[28] Roy, S., Morisi, S., Singh, N. N. \& Valle, J. W. F. The Cabibbo angle as a universal seed for quark and lepton mixings. Phys. Lett. B748, 1-4 (2015). 1410.3658.

[29] Morisi, S., Peinado, E., Shimizu, Y. \& Valle, J. W. F. Relating quarks and leptons without grand-unification. Phys.Rev. D84, 036003 (2011). 1104.1633.

[30] Morisi, S., Nebot, M., Patel, K. M., Peinado, E. \& Valle, J. Quark-Lepton Mass Relation and CKM mixing in an A4 Extension of the Minimal Supersymmetric Standard Model. Phys.Rev. D88, 036001 (2013). 1303.4394.

[31] Babu, K. S., Ma, E. \& Valle, J. W. F. Underlying a(4) symmetry for the neutrino mass matrix and the quark mixing matrix. Phys. Lett. B552, 207-213 (2003). hep-ph/ 0206292.

[32] Hirsch, M., Romao, J. C., Skadhauge, S., Valle, J. W. F. \& Villanova, A. Phenomenological tests of supersymmetric a4 family symmetry models of neutrino mass (2003). In preparation.

[33] Morisi, S., Forero, D., Romao, J. C. \& Valle, J. W. F. Neutrino mixing with revamped A4 flavour symmetry. Phys.Rev. D88, 016003 (2013). 1305.6774.

[34] Boucenna, M., Morisi, S., Peinado, E., Shimizu, Y. \& Valle, J. W. F. Predictive Discrete Dark Matter Model (2012). 1204.4733.

[35] Hirsch, M., Meloni, D. et al. Proceedings of the first workshop on Flavor Symmetries and consequences in Accelerators and Cosmology (FLASY2011) (2012). 1201.5525. 
[36] Morisi, S. \& Valle, J. W. F. Neutrino masses and mixing: a flavour symmetry roadmap. Fortsch.Phys. 61, 466-492 (2013). 1206.6678.

[37] King, S. F., Merle, A., Morisi, S., Shimizu, Y. \& Tanimoto, M. Neutrino Mass and Mixing: from Theory to Experiment. New J.Phys. 16, 045018 (2014). 1402.4271.

[38] Grimus, W. \& Lavoura, L. A non-standard CP transformation leading to maximal atmospheric neutrino mixing. Phys. Lett. B579, 113-122 (2004). hep-ph/ 0305309.

[39] Chen, P., Ding, G.-J., Gonzalez-Canales, F. \& Valle, J. W. F. Generalized $\mu-\tau$ reflection symmetry and leptonic CP violation. Phys. Lett. B753, 644-652 (2016). 1512 . 01551.

[40] Chen, P., Ding, G.-J., Gonzalez-Canales, F. \& Valle, J. W. F. Classifying CP transformations according to their texture zeros: theory and implications. Phys. Rev. D94, 033002 (2016). 1604.03510 .

[41] Boucenna, S. M., Fonseca, R. M., Gonzalez-Canales, F. \& Valle, J. W. F. Small neutrino masses and gauge coupling unification. Phys. Rev. D91, 031702 (2015). 1411.0566.

[42] Deppisch, F. F., Hati, C., Patra, S., Sarkar, U. \& Valle, J. W. F. 331 Models and Grand Unification: From Minimal SU(5) to Minimal SU(6). Phys. Lett. B762, 432-440 (2016). 1608 . 05334.

[43] King, S. F., Leontaris, G. K. \& Ross, G. G. Family symmetries in F-theory GUTs. Nucl. Phys. B838, 119-135 (2010). 1005.1025.

[44] Reig, M., Valle, J. W. F. \& Vaquera-Araujo, C. A. Unifying left-right symmetry and 331 electroweak theories. Phys. Lett. B766, 35-40 (2017). 1611.02066.

[45] Reig, M., Valle, J. W. F. \& Vaquera-Araujo, C. A. Three-family left-right symmetry with low-scale seesaw mechanism (2016). 1611.04571.

[46] Deppisch, F. F., Desai, N. \& Valle, J. W. F. Is charged lepton flavour violation a high energy phenomenon? Phys.Rev. D89, 051302(R) (2014). 1308.6789.

[47] Queiroz, F. S., Siqueira, C. \& Valle, J. W. F. Constraining Flavor Changing Interactions from LHC Run-2 Dilepton Bounds with Vector Mediators. Phys. Lett. B763, 269-274 (2016). 1608.07295.

[48] Chikashige, Y., Mohapatra, R. N. \& Peccei, R. D. Are there real goldstone bosons associated with broken lepton number? Phys. Lett. B98, 265 (1981).

[49] Schechter, J. \& Valle, J. W. F. Neutrino Decay and Spontaneous Violation of Lepton Number. Phys. Rev. D25, 774 (1982).

[50] Joshipura, A. S. \& Valle, J. W. F. Invisible Higgs decays and neutrino physics. Nucl. Phys. B397, 105-122 (1993).

[51] Bonilla, C., Valle, J. W. F. \& Romao, J. C. Neutrino mass and invisible Higgs decays at the LHC. Phys. Rev. D91, 113015 (2015). 1502.01649.

[52] Bonilla, C., Romao, J. C. \& Valle, J. W. F. Electroweak breaking and neutrino mass: invisible Higgs decays at the LHC (type II seesaw). New J. Phys. 18, 033033 (2016). 1511 . 07351. 
[53] Abdallah, J. et al. Searches for invisibly decaying Higgs bosons with the DELPHI detector at LEP. Eur. Phys. J. C32, 475-492 (2004). hep-ex/ 0401022.

[54] Aad, G. et al. Search for Invisible Decays of a Higgs Boson Produced in Association with a Z Boson in ATLAS. Phys. Rev. Lett. 112, 201802 (2014). 1402.3244.

[55] Bonilla, C., Fonseca, R. M. \& Valle, J. W. F. Vacuum stability with spontaneous violation of lepton number. Phys. Lett. B756, 345-349 (2016). 1506.04031.

[56] Bonilla, C., Fonseca, R. M. \& Valle, J. W. F. Consistency of the triplet seesaw model revisited. Phys. Rev. D92, 075028 (2015). 1508.02323.

[57] Addazi, A., Valle, J. W. F. \& Vaquera-Araujo, C. A. String completion of an $\mathrm{SU}(3)_{\mathrm{c}} \otimes \mathrm{SU}(3)_{\mathrm{L}} \otimes \mathrm{U}(1)_{\mathrm{X}}$ electroweak model. Phys. Lett. B759, 471-478 (2016). 1604.02117.

[58] Reig, M., Valle, J. W. F. \& Vaquera-Araujo, C. A. Realistic $S U(3)_{c} \otimes S U(3)_{L} \otimes U(1)_{X}$ model with a type II Dirac neutrino seesaw mechanism. Phys. Rev. D94, 033012 (2016). 1606.08499.

[59] Randall, L. \& Sundrum, R. A Large mass hierarchy from a small extra dimension. Phys. Rev. Lett. 83, 3370-3373 (1999). hep-ph/9905221.

[60] Chen, P. et al. Warped flavor symmetry predictions for neutrino physics. JHEP 01, 007 (2016). 1509.06683.

[61] Pasquini, P., Chulia, S. C. \& Valle, J. W. F. Neutrino oscillations from warped flavor symmetry: predictions for long baseline experiments T2K, NOvA and DUNE (2016). 1610.05962.

[62] Ade, P. A. R. et al. Planck 2015 results. XIII. Cosmological parameters. Astron. Astrophys. 594, A13 (2016). 1502.01589.

[63] Boucenna, S. M., Morisi, S., Shafi, Q. \& Valle, J. W. F. Inflation and majoron dark matter in the seesaw mechanism. Phys. Rev. D90, 055023 (2014). 1404.3198.

[64] Berezinsky, V. \& Valle, J. W. F. The KeV Majoron as a dark matter particle. Phys. Lett. B318, 360-366 (1993). hep-ph/ 9309214.

[65] Lattanzi, M. \& Valle, J. W. F. Decaying warm dark matter and neutrino masses. Phys. Rev. Lett. 99, 121301 (2007). arXiv:0705.2406 [astro-ph].

[66] Bazzocchi, F. et al. X-ray photons from late-decaying Majoron dark matter. JCAP 0808, 013 (2008). 0805.2372 .

[67] Lattanzi, M. et al. Updated CMB, X- and gamma-ray constraints on Majoron dark matter. Phys.Rev. D88, 063528 (2013). 1303.4685. 